Internist 2009 · 50:5-6

DOI 10.1007/s00108-008-2276-8

Online publiziert: 20. Dezember 2008

(c) Springer Medizin Verlag 2008

M. Reincke ${ }^{1} \cdot$ H. Lehnert ${ }^{2} \cdot$ R.E. Kolloch ${ }^{3}$

${ }^{1}$ Medizinische Klinik Innenstadt, Klinikum der Universität München

2 1. Medizinische Klinik, Klinikum der Universität zu Lübeck

${ }^{3}$ Klinik für Innere Medizin, Evangelisches Krankenhaus Bielefeld

\title{
Therapierefraktäre und sekundäre arterielle Hypertonie
}

European Society of Hypertension orientiert. Gemeinsam ist allen 3 Leitlinien, dass dem Thema der therapieresistenten Hypertonie und ihren sekundären Ursachen nur sehr geringer Raum eingeräumt wird. So umfasst der entsprechende Abschnitt in der DHL-Leitlinie 1 von 99 Seiten. Dies mag einerseits an der Zielgruppe der Leitlinien liegen, die sich in erster Linie an Allgemeinmediziner richtet, andererseits an der relativen Seltenheit sekundärer Ursachen. Die Relevanz sekundärer Ursachen darf allerdings auf keinen Fall unterschätzt werden. Nach neueren Untersuchungen ist die therapierefraktäre Hypertonie, definiert als Nichterreichen des Zielblutdrucks von $<140 / 90 \mathrm{mmHg}$ trotz Behandlung mit 3 ausreichend dosierten Antihypertensiva inklusive eines Diuretikums, von quantitativ zunehmender Bedeutung: etwa $4 \%$ aller Hypertoniker in allgemeinmedizinischen Praxen weisen eine Therapieresistenz auf. In Hypertonie-Spezialambulanzen liegt diese Zahl naturgemäß deutlich höher bei bis $\mathrm{zu} 15 \%$.

cellence (NICE) Leitlinien zum „management of hypertension in adults in primary care" [1] herausgegeben. 2007 folgte die European Society of Hypertension und die European Society of Cardiology mit viel beachteten und diskutierten neuen Leitlinien für die Behandlung der arteriellen Hypertonie [3]. Zum 01.06.2008 ließ nun die Deutsche Hochdruckliga eine aktualisierte Leitlinie zur Diagnostik und Therapie der arteriellen Hypertonie veröffentlichen [2], die sich an den Guidelines der

\section{( Therapierefraktäre Hypertonie ist definiert als Nichterreichen des Zielblutdrucks von $<140 / 90$ trotz Behandlung mit 3 ausreichend dosierten Antihypertensiva inkl. eines Diuretikums}

In der einleitenden Arbeit dieses Hefts wird ein Überblick über die vorhande- ne Literatur zu Epidemiologie und Ätiologie der therapieresistenten Hypertonie gegeben. Ursächlich müssen hier zunächst vor allem die „Klassiker“ der scheinbaren Therapieresistenz ausgeschlossen werden: Patienten-Noncompliance, inadäquate Blutdrucktherapie und Weißkittelhypertonie. Der echten Therapieresistenz liegt häufig ein deutliches Übergewicht, ein höheres Lebensalter, ein Schlafapnoesyndrom, eine Niereninsuffizienz oder Medikamenteninterferenzen zugrunde.

Der Artikel von Eric Born-Frontsberg und Marcus Quinkler stellt die aktuelle Literatur zum primären Hyperaldosteronismus zusammen. Der häufig normokaliämische M. Conn ist nach neueren Untersuchungen mit einer Prävalenz von $12 \%$ in spezialisierten Hypertonieambulanzen [4] eine der häufigsten sekundären Hypertonieursachen. In Deutschland könnten rechnerisch bis zu 2,5 Mio. Menschen durch einen primären Hyperaldosteronismus betroffen sein. Hauptproblem bleibt das Fehlen einer einfachen und zuverlässigen Screeninguntersuchung, mit der dieses Krankheitsbild mit vertretbaren Kosten und ausreichender Spezifität diagnostiziert werden kann.

Die Arbeit von Reisch et al. beschäftigt sich mit dem Phäochromozytom, welches aufgrund seines variablen klinisches Erscheinungsbilds und der unbehandelt deletären Kreislaufkomplikationen immer noch eine Herausforderung in Diagnostik und Therapie darstellt. Neu ist, das bei einem Viertel aller Phäochromozytompa- 
tienten Keimbahnmutationen vorliegen. Deshalb sollte auch bei scheinbar sporadischen Tumoren eine risikoadaptierte Mutationsanalyse durchgeführt werden.

Die Häufigkeit eines Cushing-Syndroms als Ursache einer Hypertonie ist sehr gering und wird mit maximal o,2\% angegeben. Allerdings muss bei Vorliegen einer therapieresistenten Hypertonie immer ein Cushing-Syndrom durch sorgfältige klinische Untersuchung und ggf. biochemische Testung ausgeschlossen werden. Wie dieses zu erfolgen hat, wird in der Arbeit von Dodt et al. in diesem Heft dargestellt.

Der letzte Beitrag in diesem Heft von A. Voiculescu und L.C. Rump befasst sich mit der Diagnostik und Therapie der Nierenarterienstenose. Drei prospektive, randomisierte, kontrollierte Studien konnten bisher keine eindeutige Überlegenheit einer Intervention mittels Ballondilatation zur Beseitigung der Stenose im Vergleich zu einem konservativ-medikamentösen Vorgehen hinsichtlich Blutdruckkontrolle oder kardiovaskulärer Endpunkte zeigen. Die geeigneten Patienten für eine Katheterintervention müssen deshalb sehr sorgfältig selektioniert werden.

Zusammengefasst hoffen wir Ihnen mit diesem Heft einen aktuellen und praxisrelevanten Überblick über dieses spannende Gebiet der Hypertoniediagnostik und -therapie verschaffen zu können.

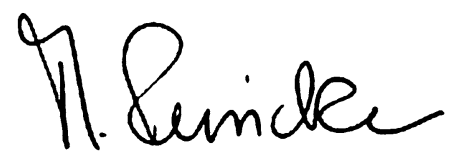

Prof. Martin Reincke

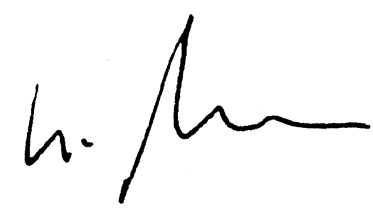

Prof. Hendrik Lehnert

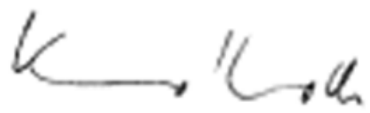

Prof. Rainer E. Kolloch

\section{Korrespondenzadresse}

\section{Prof. Dr. M. Reincke}

Medizinische Klinik Innenstadt,

Klinikum der Universität München

Ziemssemstraße 1, 80336 München

Martin.Reincke@med.uni-muenchen.de

Interessenkonflikt. Der korrespondierende Autor gibt an, dass kein Interessenkonflikt besteht.

\section{Literatur}

1. (2007) Summary of the 2007 European Society of Hypertension (ESH) and European Society of Cardiology (ESC) guidelines for the management of arterial hypertension. Vasc Health Risk Manag 3: 783-795

2. http://www.hochdruckliga.info

3. http://www.nice.org.uk/nicemedia/pdf/CG034NICEguideline.pdf

4. Rossi GP, Bernini G, Desideri G et al. (2006) Renal damage in primary aldosteronism: results of the PAPY study. Hypertension 48: 232-238

\section{Ausschreibung: Wilhelm P. Winterstein-Preis 2009 der Deutschen Herzstiftung e.V.}

Die Deutsche Herzstiftung vergibt im Jahr 2009 ein weiteres Mal den Wilhelm P. Winterstein- Preis, dotiert mit 10000 Euro. Ausgezeichnet wird eine wissenschaftliche Arbeit auf dem Gebiet der Herz-KreislaufErkrankungen, bevorzugt aus einem patientennahen Forschungsbereich.

Die Arbeit darf einen Gesamtumfang von 25 Seiten nicht überschreiten und in dieser Form noch nicht veröffentlicht worden sein. Ihr ist eine Zusammenfassung in deutscher Sprache voranzustellen. Teilnahmeberechtigt sind Ärztinnen und Ärzte aus Deutschland.

Die Bewerbungsunterlagen mit tabellarischem Lebenslauf sind in dreifacher Ausfertigung bis spätestens 23. Februar 2009 (Poststempel) zu senden an: Deutsche Herzstiftung e.V., Vogtstraße 50, 60322 Frankfurt am Main. Sind Co-Autoren an der Arbeit beteiligt, ist deren Einverständniserklärung zur Bewerbung sowie Angaben über deren jeweilige Arbeitsanteile beizufügen.

Die Preisverleihung erfolgt im Rahmen der Mitgliederversammlung der Deutschen Herzstiftung im Juni 2009 in Frankfurt am Main. Weitere Informationen sind zu erhalten über Christine Koch unter der Telefonnummer (069) 9551 28-128 oder der Homepage www.herzstiftung.de der Deutschen Herzstiftung zu entnehmen.

Quelle: Deutsche Herzstiftung (Frankfurt/ Main). 